\title{
OPTICAL MEMS: LEGACY OF THE TELECOM BOOM
}

\author{
Joseph E. Ford \\ Electrical \& Computer Engineering, University of California San Diego \\ La Jolla, CA 92093-0407
}

\begin{abstract}
From 1997 to 2002 tens of billions of dollars were invested in optical MEMS technology for telecommunications, creating products just in time for a nearly complete market collapse. With the subsequent closure of R\&D efforts and many entire companies, has this new technology been lost? In fact, many MEMS-based components survived the collapse and are selling into a slowly recovering market. More importantly, the technology developed is available for new applications in communications and other areas. In this presentation I will review optical MEMS technology developed, provide a status report on surviving components, and describe one example of how a device created for a specific application (a broad wavelength variable attenuator) has been modified to a completely different application in free-space optical communications (a retro-reflecting data modulator).
\end{abstract}

\section{OPTICAL MEMS AND TELECOM}

Any optical effect demonstrated with bulk components can be implemented, typically on a reduced physical scale, using optical MEMS fabrication. These basic functions are illustrated in Figure 1, which shows the four major categories of effects: movable first surface mirrors (analog or digital tilt mirrors on one or two axis), movable absorbing or refractive surfaces, tunable dielectric multilayer etalons (spectral phase and amplitude filters), translation stages (moving waveguides and shutters), as well as diffractive structures (variable phase delay or grating pitch).

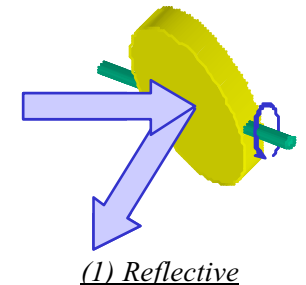

(analog tilt mirror shown)

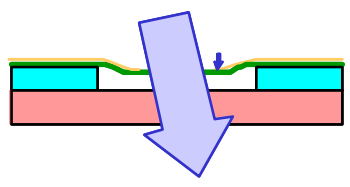

(3) Interferometric

(MARS modulator shown)

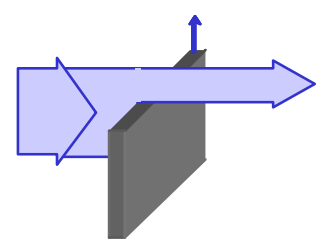

(2) Absorptive / Refractive (digital shutter shown)

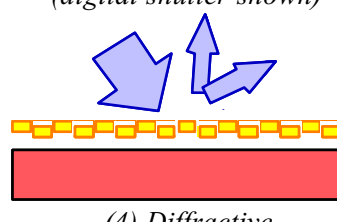

(4) Diffractive

(grating light valve shown)
Figure 1. Fundamentally different types of optical MEMS devices.

The initial driving application for Optical MEMS devices was Texas Instruments' well-known DMD (digital mirror device) projection display, a 2D array of digital tilting micromirrors fabricated on a silicon VLSI drive circuit [1]. DMD displays are becoming the dominant technology for commercial and even consumer projectors, and optical MEMS devices have now been developed for applications including remote pressure and acceleration sensors, biotechnology, and adaptive optics. Still, the greatest driver for optical MEMS technology in terms of total investment has been telecommunications.

Early optical MEMS components intended for telecom applications were viewed with considerable skepticism; the technology was considered unproven and far off the consensus path of waveguide integrated optics. A reflective data modulator demonstrated in 1994 for fiber-to-the-home networks [2] may have been the first practical MEMS device for single mode fiber. This device was not developed into a successful product, but it was followed by a series of optical MEMS switches and filters for wavelength-multiplexed signals which demonstrated the broad applicability of optical MEMS components for optical communications. References [3] - [9] provide some examples.

By 1999, with increasing pressure to meet market demand for high-capacity networks, well-funded optical MEMS component efforts were under way at established and start-up companies. Many applications could be addressed with a range of technologies, but the large port-count provisioning switch (e.g., the Lucent "LambdaRouter" [10]) was a 'must-have' telecommunications component that simply could not be constructed with any other available technology. Optical MEMS was perceived as critical-path technology for high-capacity optical networks.

Demand for communications bandwidth had been greatly overestimated. The market peaked in mid 2000, and by 2002 technology investment was substantially over. Relatively few of the companies founded during the investment boom (by some estimates more than 3000 in total) survive to date. But though this process, a wide range of optical MEMS components were taken from laboratory demonstrations to fully packaged components, proven to meet critical Telcordia reliability tests, and built into fielded systems carrying live communications traffic. Despite the considerable disruption, a wide range of MEMS-based components are still commercially available, and have make the transition to a "bread and butter" technology.

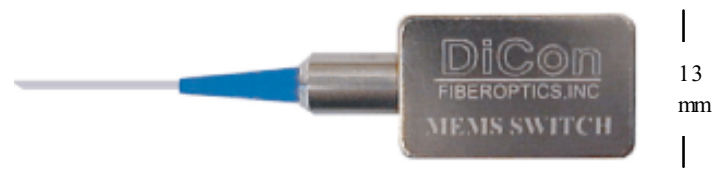

Figure 2. A simple 1x2 protection switch is one example of the optical MEMS products now commonplace in telecom systems.

\section{TELECOM 'SURVIVORS'}

The single best indication of this acceptance is the use of MEMS devices for protection switches, which are $1 \times 2$ and $2 \times 2$ switches used to direct traffic around faulty transmission systems, as for example due to a line cut. MEMS switches were shown to be more reliable than the electromagnetically-actuated (bulk optic component) opto-mechanical switches previously used, in addition to being smaller and more power efficient. Two current products 
are JDSU's latching $2 \times 2$ switch, which is based on a SOI combdrive actuator [11] and DiCon 1x2 switch with variable attenuation, which s based on an analog electrostatic tilt mirror. The DiCon switch pictured in Figure 1 has an insertion loss of $0.8 \mathrm{~dB}$, switches in under $5 \mathrm{~ms}$, requires only $15 \mathrm{~V}$ drive, and is guaranteed to operate over 100 million cycles [12].

Optical MEMS companies started during the boom have also survived to offer high-performance products to the slowly recovering component market. Beam-steering optical crossconnects based on two-dimensional arrays of analog tilt mirrors are available from Glimmerglass, which provides an 64x64 switch, and Calient Networks, which makes up to 256x256 switches [13]. Chromux offers $1 \times 2$ protection switch arrays, as well as scanning Fabry-Perot wavelength monitors.

Another important class of optical MEMS components integrates diffraction gatings to perform spectral demultiplexing onto the MEMS device to enable wavelength-selective filtering and switching. Dynamic spectral equalizers and wavelength-blocking switches are offered by LightConnect and Polychromix, while more general wavelength switching $[4,14]$ is offered by Capella Photonics. Figure 3 shows a representative sample.

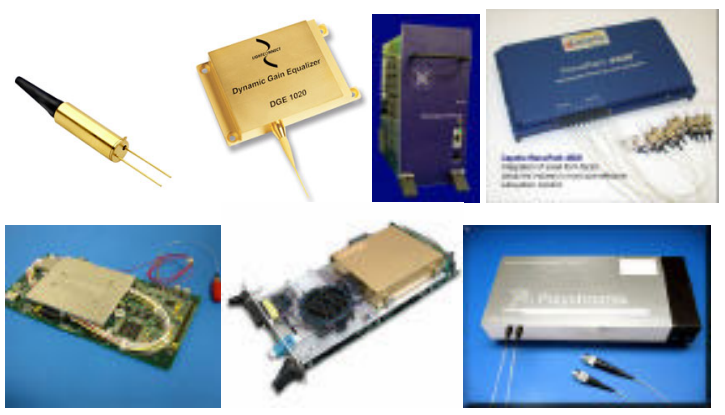

Figure 3. Examples of currently available telecom products, clockwise from upper left: LightConnect variable attenuator and dynamic gain equalizer, Glimmerglass $64 x 64$ switch, Capella Photonics wavelength selective switch, Polychromix wavelength blocker, Chromux protection switch array and wavelength monitor

\section{OPTICAL MEMS DEVICE PROGRESS}

These basic functions have been demonstrated with various device structures, some of which were never publicized and which remain 'trade secrets' of technology holding companies. Device packaging and processing techniques are particularly difficult to obtain. However, some of the device technology has since been published in the open literature.

One good example is the feedback position control of electrostatic tilt mirrors used by Tellium in their modular crossconnect system [15]. Normally such mirrors are operated in a safe region, roughly half the full angular range, to avoid catastrophic snapdown as the voltage required to maintain a given angle quickly decreases. Tellium used a nonlinear controller with a novel technique for torque-to-voltage conversion [16] in combination with classic linear controller techniques with optical full-state feedback, state estimator, and reference input with feed-forward. They achieved stable angular positioning accuracy of $<120$ microradians over the full $+/-8^{\circ}$ mechanical range of the mirrors, right up to the point of edge touchdown (Figure 4).
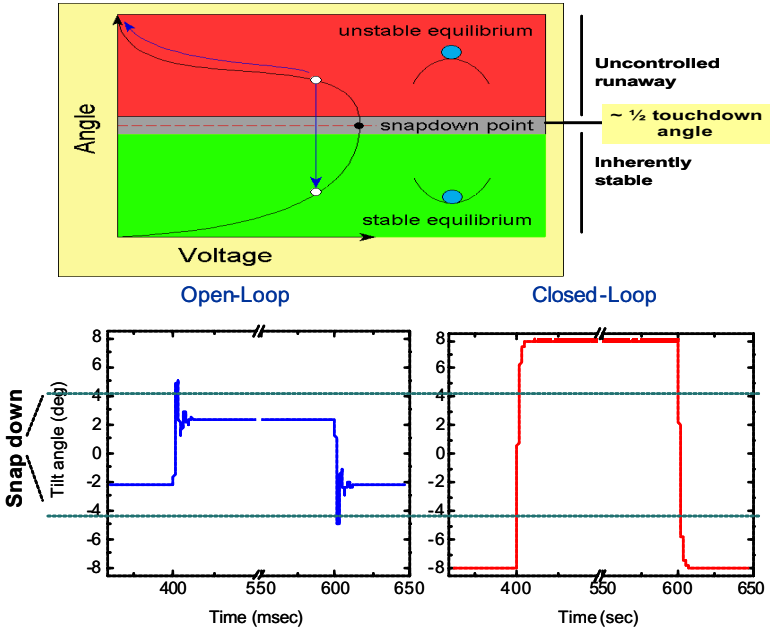

Figure 4. Control of electrostatic mirrors through snapdown using optical feedback on the angular position $[15,16]$.

Another area of technology development critical for low insertion loss optical MEMS devices is the accurate control of mirror (and etalon) curvature. Some of these efforts are widely available as improved tolerances and process yields from MEMS foundries (e.g., mirrors with $>1 \mathrm{~m}$ radius of curvature, $10-100 \mathrm{x}$ improved over initial efforts). Also, basic work on thermal dependence of mirror curvatures has also been conducted at public universities including the University of Minnesota [17].

\section{TECHNOLOGY RE-DEPLOYMENT: AN EXAMPLE}

The basic technology development will have far-ranging impact on applications that have nothing to do with optical fiber networks. One example of this re-use is an on-going research project at UCSD involving the modification of a telecom-derived MEMS device for free-space (mobile) communications.

Fast, electrically-controlled optical fiber attenuators were needed for transient suppression in long-haul fiber communications. In 1996, a Bell Labs research project involved a novel MEMS wavefront modulator for this application [19]. A membrane reflector was formed over a hexagonal array of cavities so that electrostatic force could deform the normally flat reflector into a shape like the surface of a golf ball. A collimated beam illuminating the modulator is efficiently coupled into the output fiber, but when voltage is applied, a controlled fraction of light is aberrated away from the optical fiber core and discarded. The primary advantage of this device was that the mechanical response time was determined not by the overall optical aperture, but by the diameter of the individual membrane aperture.

This attenuator was successfully demonstrated using a variation on MARS etalon optical modulator process [2], where a silicon nitride membrane is held over the silicon substrate by a phosphor-silicate glass sacrificial layer. The only process change needed was to coat a uniform layer of gold over the top of the device. This device concept was independently conceived and demonstrated at the University of Delft in 1999 [20].

The new application of this device involved orner-cube retroreflectors (CCR), which are basically three mirrors are right angles to each other to form a hollow cube that faithfully reflects an incident optical beam towards it's point of origin. CCRs are used 
to self-align free-space optical signals, as for example in a surveyor's rangefinder. A retro modulator, a retroreflector with an electrically-controlled reflectivity, can communicate data signals back to a laser source without needing to align a separate laser transmitter at the remote node.

Retro modulators can block the beam (amplitude modulation), but it is equally effective to phase-modulate the back-propagating wavefront so that the return signal is dispersed and does not arrive at the remote detector. A MEMS retro modulator demonstrated by UC Berkeley [18] for their "Smart Dust" project used two fixed micro-mirrors assembled over a tilting MEMS mirror, so that the CCR angle could be switched away from $90^{\circ}$. The resulting device had a $1 \mathrm{~mm}$ aperture and a $2 \mathrm{kHz}$ response. However, our goal was fabricate a retro modulator with a substantially larger aperture (10 to $25 \mathrm{~mm}$ ) suitable for long-range (multi-km) optical communications, and operate the device at much higher speeds $(>100 \mathrm{KHz})$ than possible moving a single large mirror. The Bell Labs attenuator device was ideal for this application.

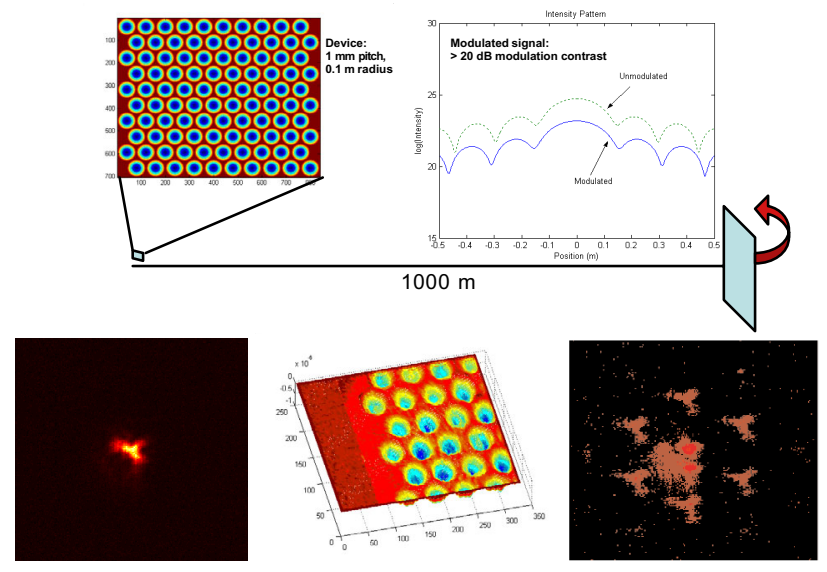

Figure 5. Simulation (top) and experimental results from the membrane modulator used for free-space optical communications.

A theoretical calculation of the wavefront propagating from a membrane modulator with $1 \mathrm{~mm}$ pixels with a $100 \mathrm{~cm}$ radius of curvature is shown at the top of Figure 5. More than $20 \mathrm{~dB}$ contrast is achieved over a wide range of angles, distances, and operating wavelengths. The lower three images are preliminary experimental results from a fabricated device. They show the optical far-field signal reflected from an undeflected modulator, the surface profile from the actuated device, and the dispersed signal reflected from the actuated modulator.

\section{CONCLUSION}

The telecom boom yielded a wealth of optical MEMS technology which, in addition to serving it's original purpose of telecom components, is available for new applications in sensing and communications. The next wave of research and developmenthopefully less turbulent than the last - has already begun.

\section{ACKNOWLEDGEMENTS}

Research conducted at UCSD's Photonics Systems Integration Laboratory is supported by a grant from Cubic Corporation. The experimental results shown are the work of graduate student researcher Trevor Chan.

\section{REFERENCES}

1. P. Van Kessel et al, "A MEMS-based projection display," IEEE Proceedings 86(8), pp. 1687-1704 (1998).

2. K.W. Goossen, J. A. Walker, S. C. Arney, "Silicon modulator based on mechanically-active anti-reflection layer with $1 \mathrm{mbit} / \mathrm{sec}$ capability for fiber-in-the-loop applications," IEEE Photonics Technology Letters 6(9), pp. 1119 - 1121 (1994).

3. M. Wu, E. Vail, G. Li, and C. Chang-Hasnain, Widely and continuously tunable micromachined resonant cavity detector with wavelength tracking," IEEE Phot. Tech. Lett., 8 pp.98-100 (1996).

4. L. Lin, E. Goldstein, R. Tkach, "Free-space micromachined optical switches with sub-ms switching time for large-scale optical crossconnects," IEEE Phot. Tech. Letters 10 pp. 525-527 (1998).

4. J. Ford, J. Walker, "Dynamic spectral power equalization using micro-opto-mechanics," IEEE Phot. Tech. Lett. 10(10), (1998).

5. J. Ford et al, "Micromechanical fiber-optic attenuator with 3 microsecond response," IEEE J. Lightwave Tech. 16(9), (1998).

6. P. Tayebati, P. Wang, D. Vakhshoori, and R. Sacks, "Widely tunable Fabry-Perot filter using $\mathrm{Ga}(\mathrm{Al}) \mathrm{As} / \mathrm{AlOx}$ deformable mirrors" Optical Society of America Conference on Optical Fiber Communications 1998, pp. 22-27 (1998).

7. J. Ford, J. Walker, V. Aksyuk and D. Bishop, "Wavelength add/drop switching using tilting micromirrors," IEEE J. Lightwave Tech. 17(5), pp. 904-911, (1999).

8. C. Marxer and N. de Rooij, "Micro-opto-mech. 2 x 2 switch for single-mode fibers based on plasma-etched silicon mirror and electrostatic actuation" J. Lightwave Tech.17(1), pp. 2-6, (1999).

9. P. Hagelin et al, "Scalable optical cross-connect switch using micromachined mirrors," IEEE Phot. Tech. Lett. 12(7), pp. 882-884 (2000).

10. D. T. Neilson et al, "Fully provisioned $112 \times 112$ micromechanical optical crossconnect with $35.8 \mathrm{~Tb} / \mathrm{s}$ demonstrated capacity," OSA Conference on Optical Fiber Communications 2000, postdeadline paper pp. $202-204,(2000)$.

11. B. Hichwa et al, "A Unique Latching 2x2 MEMS Fiber Optics Switch”, IEEE International Conf. on Optical MEMS, (2000).

12. DiCon Fiber Optics product datasheet, http://www.diconfiberoptics.com/products/scd0061/index.htm

13. J. Xuezhe et al, "Three-dimensional MEMS photonic crossconnect switch design and performance," IEEE Journal of Selected Topics in Quantum Electronics, 9(2), pp. 571-578 (2003).

14. D. Marom et al, "Wavelength-selective $1 \times 4$ switch for 128 WDM channels at $50 \mathrm{GHz}$ spacing," Optical Society of America Conference on Optical Fiber Communications, Paper FB7, 2002

15. J. Dadap et al, "Modular MEMS-based optical cross-connect with large port-count," IEEE Photonics Technology Letters, 15(12), pp. 1773 - 1775 (2003)..

16. I. Brener et al, "Nonlinear servo control of MEMs mirrors and their performance in a large port-count optical switch," $O S A$ Conference on Optical Fiber Communications pp. 383-387 (2003).

17. K. Cao, W. Liu and J. Talghader, "Curvature compensation in micromirrors with high-reflectivity optical coatings," Journal of MEMS 10(3), pp. $409-417$ (2001).

18. L. Zhou et al, "Corner-cube retroreflectors based on structureassisted assembly for free-space optical communication, “ IEEE J. MEMS 12(3), pp. 233-242, 2003.

19. J. Ford and J. Walker, "Technique for modulating optical signals in optical communications," United States Patent number 5,796,880, filed November 1996.

20. S. Sakarya, G. Vdovin and P. Sarro, "Micromachined SLM based on pixelated reflective membranes", SPIE Proc. 3760, November 1999. 